\title{
USING 3D VISION FOR THE DIAGNOSIS AND TREATMENT OF AMBLYOPIA IN YOUNG CHILDREN*
}

\author{
Angelo Gargantini \\ Dip. di Ingegneria Informatica e Metodi Matenatici, Università di Bergamo, Viale Marconi 5, Dalmine, Italy \\ angelo.gargantini@unibg.it
}

\begin{abstract}
Keywords: Vision rehabilitation, computer aided vision therapy, amblyopia, 3D vision
Abstract: The 3D4Amb project aims at developing a system based on the NVIDIA 3D Vision for the diagnosis and treatment of amblyopia in young children. It exploits the active shutter technology to provide binocular vision, i.e. to show different images to the amblyotic (or lazy) and the normal eye. It would allow easy diagnosis of amblyopia and its treatment by means of interactive games or other entertainment activities. It should not suffer from the compliance problems of the classical treatment, it is suitable to domestic use, and it could at least partially substitute occlusion or patching of the normal eye.
\end{abstract}

\section{INTRODUCTION}

Amblyopia, otherwise known as 'lazy eye', is reduced visual acuity that results in poor or indistinct vision in an eye that is otherwise physically normal, or out of proportion to associated structural abnormalities. It may exist even in the absence of any detectable organic disease. Typically amblyopia is present in only one eye and is generally associated with a squint or unequal lenses in the prescription spectacles. This low vision is not correctable (or only partially) by glasses or contact lenses.

There exist several causes of amblyopia. Anything that interferes with clear vision in either eye during the critical period (birth to 6 years of age) can result in amblyopia. The most common causes of amblyopia are constant strabismus (constant turn of one eye), anisometropia (different vision/prescriptions in each eye), and/or blockage of an eye due to trauma, lid droop, etc. If one eye sees clearly and the other sees a blur, the good eye and brain will inhibit the eye with the blur. The brain, for some reason, does not fully acknowledge the images seen by the amblyopic or lazy eye. Thus, amblyopia is a neurologically active process. The inhibition process (suppression) can result in a permanent decrease of the vision in that eye that can not be corrected with glasses, lenses, or surgery. This condition affects $2-3 \%$ of the population, which equates to conservatively around 10 million people under the age of 8 years worldwide.

Amblyopia is currently treated by wearing an ad-

\footnotetext{
${ }^{1}$ This work is partially supported by NVIDIA corp. under the Professor Partnerships program
}

hesive patch over the non-amblyopic eye for several hours per day, over a period of several months. This treatment can require up to 400 hours in total to be effective (Cleary, 2000). This conventional patching or occlusion treatment for amblyopia often gives disappointing results for several reasons: it is unpopular, prolonged, and it can sometimes make the squint worse because it disrupts whatever fusion there is. These issues frequently results in poor or noncompliance and since the success of patching depends on compliance, it performs on average very poorly. The treatment by itself works well, but it is often abandoned because it is too much trouble to take. Very often, children are averse to wearing a patch and parents found occlusion difficult to implement (Dixon-Woods et al., 2006). For this reason, the orthoptists and ophthalmologists are continuously looking for a more acceptable solution to the problem, i.e. an effective treatment that is also complied with and so really works (Gregson, 2002).

\subsection{Computer based treatment of amblyopia}

In the last years, several research groups have experimented treatment of amblyopia by exploiting and adapting information technologies. There exist several Personal Computer (PC) based software programs that allow vision training but they still require patching of the good eye. The most original system which does not require patching, is described in the following.

The VIRART group at the University of Nottingham has developed a novel virtual-reality (VR)-based display system which avoids occlusion of the nonam- 


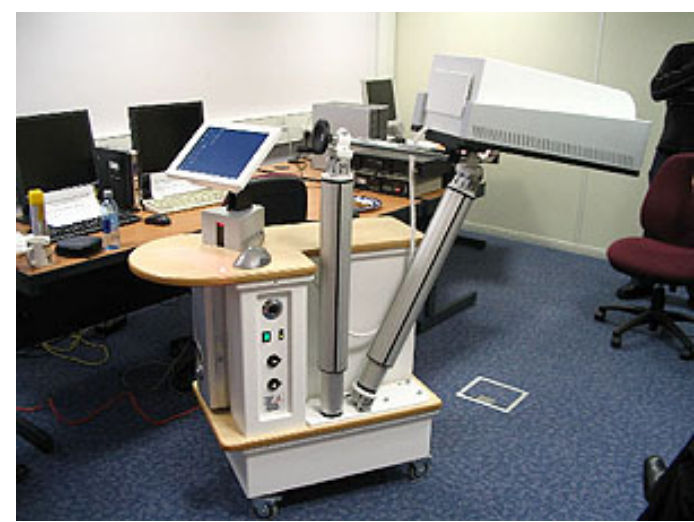

Figure 1: The I-BiT ${ }^{\mathrm{TM}}$ system. Copyright University of Nottingham, printed with permission.

blyopic eye and facilitates the treatment of amblyopia with both eyes stimulated simultaneously (Eastgate et al., 2005). This system is called I-BiT ${ }^{\mathrm{TM} 2}$. The $\mathrm{I}-\mathrm{BiT}^{\mathrm{TM}}$ system itself consists of a viewer which is linked to a PC. The PC has a standard monitor for the clinician while the viewer allows binocular vision. Several types of viewers were tested, like binocular headsets and several types of viewers called "cyberscopes" similar to that shown in Figure 1.

This system incorporates adapted VR technology and specially written software providing interactive $2 \mathrm{D}$ and $3 \mathrm{D}$ games and videos to the patient via a stereo (binocular) display. Treatment consists of watching video clips and playing interactive games in the clinic with specifically designed software to allow streamed binocular image presentation. The children sit in front of the viewer and play with the software designed for this kind of treatment.

An evaluation of I-BiT ${ }^{\text {TM }}$ The experiments show that this type of treatment can be efficiently employed and it performs better than the classical treatment. In (Waddingham et al., 2006), the results of the use of $\mathrm{I}_{-\mathrm{BiT}}{ }^{\mathrm{TM}}$ in six children are presented. In the case study, treatment consisted of watching video clips and playing simple interactive games with specifically designed software to allow streamed binocular image presentation via $\mathrm{I}_{-} \mathrm{BiT}^{\mathrm{TM}}$. Improvements in vision were demonstrable within a short period of time, in some children after $1 \mathrm{~h}$ of treatment. However, the proposed treatment has some limits. The kind of hardware system used makes the treatment rather costly and performable only in suitable clinic rooms under the supervision of a doctor (or at least of an adult). The cure can be performed only for a limited time and only with precise time scheduling. For these rea-

\footnotetext{
${ }^{2} \mathrm{http} / / / \mathrm{hfrg}$. nottingham.ac.uk/ibit/
}

sons, we believe that the I-BiT ${ }^{\mathrm{TM}}$ system suffers from the same problem of compliance of the patching treatment. The goal of this project is to design a system which could combine the performance of $\mathrm{I}_{-} \mathrm{BiT}^{\mathrm{TM}}$ but be more accessible and usable.

Other research works present similar approaches by using binocular head mounted display (HMD) or similar tools to allow binocular vision. At the best of our knowledge, no group has experimented the use of the stereo $3 \mathrm{D}$ vision systems for the diagnose and treatment of the amblyopia. In this paper we present a project, 3D4AMB which is based on the $3 \mathrm{D}$ vision, for the diagnosis and treatment of amblyopia in young children.

\section{USING THE 3D TECHNOLOGY FOR BINOCULAR VISION}

The main goal of this research project, called 3D4AMB, is to develop a system for the diagnosis and treatment of amblyopia, based on the binocular vision but that is accessible. With the term "accessible" we mean:

Inexpensive: the system needs to be relatively low in cost, it must be affordable by a family. To be so cheap, the system may be based on standard off the shelf technologies, which could be bought in stores open to the general public.

Friendly to use: the system needs to be friendly in its use such that the patients can use it without requiring a particular education or skill. The system may be operated autonomously by the children themselves and the intervention of an adult may be limited to initially set up the system (installation) and to start the treatment at least.

Suitable for domestic use: the system can be used at home without frequent time-consuming visits to the hospital. In this way, the timing of the treatment can be decided by the patients. It may use other domestic appliances like standard personal computers and televisions.

Easily extensible: it must be possible to easily develop new applications and programs to be added to the system. For this reason, standards and open software libraries may be used for developing the applications.

We have devised a system which has all the above characteristics and is based on the 3D technologies, although the goal is not to provide the patients with the $3 \mathrm{D}$ experience but to allow binocular vision. The classical use of a 3D system is to provide the two eyes with two different images of the same scene with a slightly offset viewing angles which correspond to the 


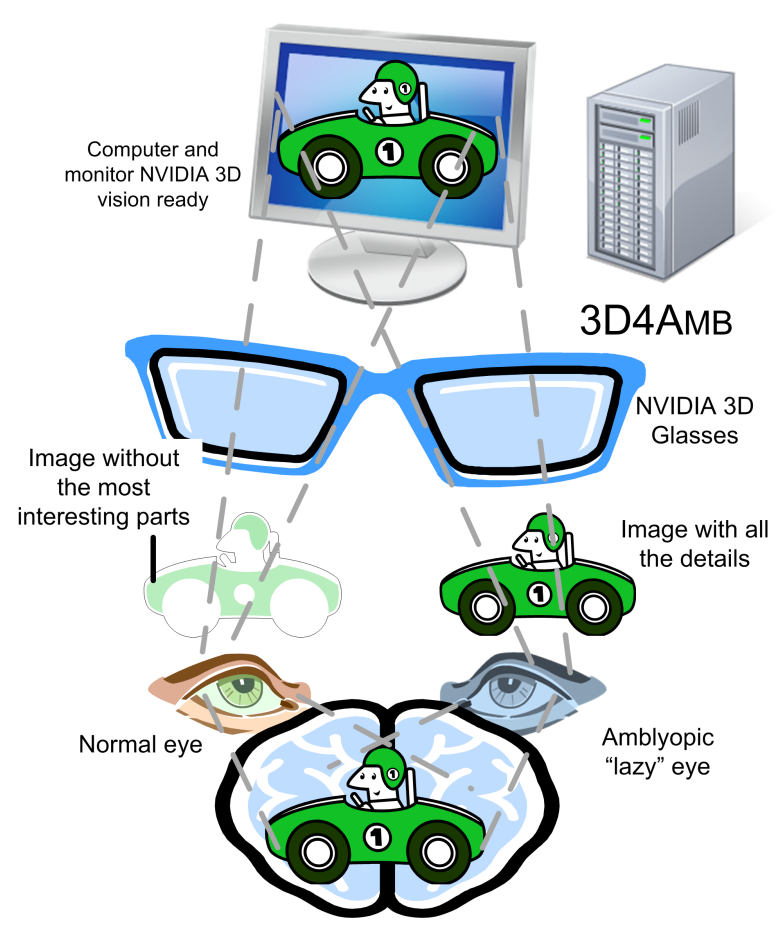

Figure 2: Basic principle of the project

different viewpoints of our left and right eye. This vision produces an illusion of real depth of the scene and it is the basis of the virtual reality. We exploit only the capability of the 3D system to send two different images to the eyes while we do not want to recreate a virtual reality.

We have already built a working prototype based on the NVIDIA $₫ 3$ D Vision ${ }^{\mathrm{TM}}$ technology, although other 3D technologies may be supported as well in the future. The NVIDIA 3D vision is based on active shutter technology which offers full image quality per eye, wide viewing angle for $3 \mathrm{D}$, excellent $2 \mathrm{D}$ Operation, and acceptable cost.

The system we have developed for 3D4AMB consists in a normal PC desktop connected to a 3D monitor (3D Vision-Ready Display). The PC must be 3D capable and have all the 3D4AMB software installed on it. The patient wears the NVIDIA active LCD shutter glasses that allow viewing a different image from the left and right eye. The scenario is depicted in Figure 2.

The basic principle of the system is that the amblyopic or 'lazy' and the normal eye are shown two different but related images. This principle can be used in practice for the treatment of amblyopia, where the amblyopic eye is shown the more interesting part of the images of the clip or of the game, while the non-amblyopic or 'good' eye is shown the less inter-

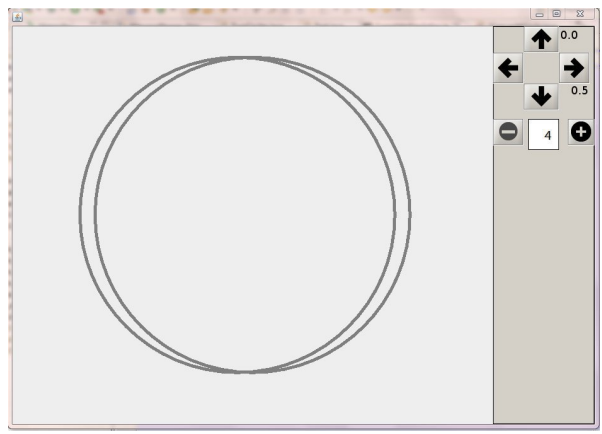

Figure 3: Diagnosis Application

esting part of the image. The content to be shown by the patient (game or image) is split by 3D4AMB in two parts, one for the right eye (the amblyopic eye in the Figure) and one for the left eye (the good eye in the Figure). The 3D4AMB software will decide what to send to both eyes depending on the type of treatment suggested by the physician. Note that the lazy eye of the child is more stimulated to work, but the non-amblyopic eye is not patched. The patient brain must join the two images to successfully see the complete image and successfully perform simple tasks in case of an interactive game. To make sure that the patient can join the two images there are a significant number of elements common to both images. Note that the final image is a bidimensional image because the goal in not to stimulate the stereo vision of the patient (at least initially).

\subsection{Use Cases of 3D4AMB}

We have designed the following use cases that model the ways we expect 3D4AMB will be used and the possible interactions with physicians and patients. We have also developed simple prototype applications to prove that the designed usage of 3D4AMB is feasible.

Diagnosis. The 3D4AMB system can be used for the screening and measurement of the amblyopia. The physician will save the parameters corresponding to the kind of amblyopia and these data will be reloaded by the 3D4AMB software used by the patient. In Figure 3 we show a simple application that permits to measure the squint between the two eyes. Two circles are shown to the patient, one for each eye. The two circles are translated until the patient sees only one circle.

Passive Image and Movie Viewing. Another use of 3D4AMB is to visualize images and clips. 3D4AMB includes an image and video viewer that is able to send two different images to both eyes. This activity, although it is passive, since it does not require any action by the child, could be performed for prolonged 


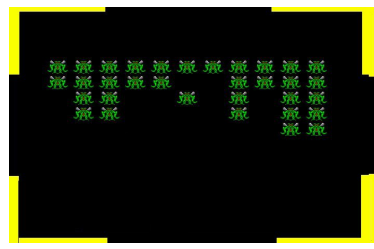

(a) Normal Eye

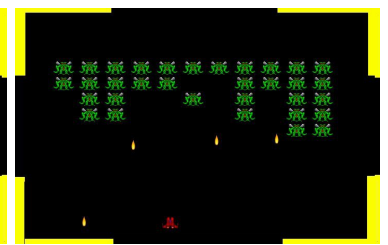

(b) Lazy Eye
Figure 4: Space Invaders Game

time and would allow to exercise the lazy eye while performing activities, like watching movies, likely to be appealing for children.

Video Games and Exercises. While patching is a passive method, other treatments which require some activity on the part of the patients are classified as $a c$ tive. Active methods are intended to enhance treatment of amblyopia in a number of ways, including increased compliance and attention during the treatment periods (due to activities that are interesting for the patient) and the use of stimuli designed to activate and to encourage connectivity between certain cortical cell types.

The most advanced and active use of 3D4AMB is the active playing with interactive games or exercises, which will stream binocular images. It is well known that video games can be very useful for visual rehabilitation (Achtman et al., 2008). In this settings, the child plays with a special video game which sends to the lazy eye all the details while the normal eye will see only a part of the game scene. To successfully complete the game the patient must use the information shown to the lazy eye (and fuse it with that shown to the normal eye). In this way, the amblyotic eye is more stimulated and the fusion encouraged. The game application can continuously monitor the success rate of the game in order to adjust the difficulty based on the real capability of the player.

We have developed a small space invaders games shown in Figure 4 in which the entire scene is presented to the lazy eye, while the normal eye does not see the spacecraft (red in the Figure) and the shots (yellow in the Figure). The player must hit the invaders (green in the Figure) firing some shots by pressing the space bar on the keyboard (or a fire button of a joystick). Some parts of the background (in yellow in the Figure) are shown to both eyes to facilitate fusion.

\subsection{Software architecture}

The 3D4AMB software is based on several components which facilitate the development of new applications. The system works only (up to now) on a PC with Microsoft Windows $\AA$ Vista 32/64-bit or Windows 7 32/64-bit Operative systems with the NVIDIA 3D Vision drivers and with Sun Microsystems Java Runtime Environment. It is based on the Java OpenGL (JOGL) which is a wrapper library that allows OpenGL to be used in the Java programming language. It is currently an independent open-source project under the BSD license and on Jadis (Java Advanced Display Infrastracture for Stereo) which provides a common interface for displaying Swing GUI components in stereo.

\section{CONCLUSIONS}

In this paper we have presented a system, 3D4AMB, for the diagnosis and treatment of amblyopia in young children which is based on 3D vision technologies. The 3D vision is exploited to allow the binocular vision, i.e. to send different images to the normal and the lazy eye, in order to exercise and stimulate the lazy eye and the brain to fuse the images in an unique view. We have presented several use cases supported by prototypes we have developed using Java and JOGL technologies for stereo viewing. The system has been devised with the goal to improve compliance with the treatment. It should not suffer from the non-compliance problems of the classical patching treatment. The children should enjoy the domestic use of 3D4AMB games and exercises, allowing prolonged therapy and the system promise to be inexpensive enough to foster its usage.

\section{REFERENCES}

Achtman, R., Green, C., and Bavelier, D. (2008). Video games as a tool to train visual skills. Restorative $\mathrm{Neu}$ rology and Neuroscience, 26(4-5):435-446.

Cleary, M. (2000). Efficacy of occlusion for strabismic amblyopia: can an optimal duration be identified? British Journal of Ophthalmology, 84(6):572-578.

Dixon-Woods, M., Awan, M., and Gottlob, I. (2006). Why is compliance with occlusion therapy for amblyopia so hard? a qualitative study. Arch Dis Child, 91(6):491494.

Eastgate, R. M., Griffiths, G. D., Waddingham, P. E., Moody, A. D., Butler, T. K. H., Cobb, S. V., Comaish, I. F., Haworth, S. M., Gregson, R. M., Ash, I. M., and Brown, S. M. (2005). Modified virtual reality technology for treatment of amblyopia. Eye, 20(3):370-374.

Gregson, R. (2002). Why are we so bad at treating amblyopia? Eye, 16(4):461-462.

Waddingham, P. E., Butler, T. K. H., Cobb, S. V., Moody, A. D. R., Comaish, I. F., Haworth, S. M., Gregson, R. M., Ash, I. M., Brown, S. M., Eastgate, R. M., and Griffiths, G. D. (2006). Preliminary results from the use of the novel interactive binocular treatment (IBiT[trade]) system, in the treatment of strabismic and anisometropic amblyopia. Eye, 20(3):375-378. 